\title{
Comparison of the effects of amrinone and sodium nitroprusside on haemodynamics, contractility, and myocardial metabolism in patients with cardiac failure due to coronary artery disease and dilated cardiomyopathy
}

\author{
P T WILMSHURST, D S THOMPSON, S M JUUL, B S JENKINS, D J COLTART, \\ $M$ M WEBB-PEPLOE \\ From the Department of Cardiology, St Thomas' Hospital, London
}

SUMMARY The effects of intravenous amrinone and sodium nitroprusside on haemodynamic indices, left ventricular contractility, and myocardial metabolism were compared in patients with cardiac failure. All patients received one dose of each drug and some received serial doses. Eight patients had dilated cardiomyopathy and six coronary artery disease, but the responses to the two drugs were independent of the aetiology of cardiac failure. Both drugs lowered left ventricular end diastolic pressure and aortocoronary sinus oxygen difference and increased cardiac index and left ventricular efficiency; these effects were dose related. Although the effects of the drugs on peripheral blood substrate concentrations were different, those on myocardial substrate metabolism were identical. Pressure derived indices of contractility in each group of patients were unaltered by either drug. After amrinone administration increases in cardiac index were related to plasma amrinone concentration, but alterations in contractility were not. In four individual patients increases in contractility were associated with alterations in plasma metabolite concentrations, which suggested that catecholamine release had occurred. For the groups of patients as a whole, however, amrinone had effects which did not differ significantly from those of the pure vasodilator, nitroprusside. There was no evidence that amrinone had a direct positive inotropic effect since no dose related changes in indices of contractile function could be established.

Amrinone (Inocor, Sterling-Winthrop) has been reported to produce considerable improvements in ventricular filling pressures and cardiac index in patients with cardiac failure during intravenous ${ }^{1}$ and oral administration. ${ }^{2}$ An increase in maximum rate of rise in left ventricular pressure $(\max \mathrm{dP} / \mathrm{dt}$ ) after intravenous administration of amrinone in some small series $^{3-5}$ has largely been attributed to a direct positive inotropic effect of the drug, although no dose response relation has been found, and a larger series ${ }^{6}$ has failed to confirm these changes in $\max \mathrm{dP} / \mathrm{dt}$ and other contractility indices. In isolated myocardium

Requests for reprints to Dr P T Wilmshurst, Department of Cardiology, St Thomas's Hospital, London SE1 7EH.

Accepted for publication 14 February 1984 from normal animals ${ }^{7}$ and in animals in which acute cardiac failure has been produced experimentally ${ }^{8}$ amrinone does indeed have positive inotropic properties. These properties, however, are not readily detectable at concentrations obtained during chronic oral treatment in patients (approximately $0.4-4 \mu \mathrm{g} / \mathrm{ml}) .{ }^{9-11}$ Furthermore, in myocardium from animals ${ }^{12}$ and patients ${ }^{13}$ with chronic cardiac failure the effect is not easily demonstrable even at considerably higher concentrations. Amrinone does, however, have powerful direct vasodilator properties in human ${ }^{13}$ and animal vessels, ${ }^{8}$ which are detectable at concentrations obtained therapeutically in man. ${ }^{13}$ Some workers have suggested that in chronic heart failure this vasodilator effect is largely responsible for the drug's beneficial effect on haemodynamic indices without it having any clinically demonstrable positive inotropic effect. ${ }^{6}$ 
Table 1 Clinical details of patients in the study

\begin{tabular}{lll}
\hline & $\begin{array}{l}\text { Dilated cardiomyopathy } \\
(\boldsymbol{n}=8)\end{array}$ & $\begin{array}{l}\text { Coronary artery disease } \\
(\boldsymbol{n}=6)\end{array}$ \\
\hline Mean (SEM) age (yr) & $38 \cdot 3(4 \cdot 1)$ & $53 \cdot 3(2 \cdot 3)$ \\
Mean (SEM) length of history of cardiac failure (months) & $13 \cdot 3(5 \cdot 4)$ & $6 \cdot 8(3 \cdot 5)$ \\
No of patients with symptoms (NYHA grade): & & - \\
II & 3 & 4 \\
III & 5 & 2 \\
IV & - & 6 \\
No of patients taking: & 8 & 4 \\
Diuretics & 2 & 2 \\
Vasodilators & 6 & 1 \\
Digoxin & - & 1 \\
Amiodarone & - & 2 \\
Aminophylline & - & $219 \cdot 7(12 \cdot 4)$ \\
Warfarin & $188 \cdot 1(11 \cdot 6)$ & $0 \cdot 15(0 \cdot 01)$ \\
LVangiographic data (mean (SEM)): & $0 \cdot 20(0 \cdot 02)$ & $339 \cdot 8(34 \cdot 0)$ \\
Normalised end diastolic volume $\left(\mathrm{ml} / \mathrm{m}^{2}\right)$ & $344 \cdot 4(24 \cdot 5)$ & \\
Ejection fraction & & \\
Mass (g) & & \\
\hline
\end{tabular}

${ }^{\star} \mathrm{p}<0.05$ between groups.

The actual mechanism by which amrinone produces its effect might be of little clinical significance but for the fact that intravenous and oral administration of the drug to patients is attended by a relatively high incidence of adverse effects. ${ }^{91415}$ Thus it is important to know if the drug has any useful properties in addition to those found with conventional vasodilators.

An understanding of the drug's mode of action is also important since a number of similar agents (for example, milrinone, Winthrop ${ }^{16}$; ARL-115, Boehringer Ingelheim ${ }^{17}$ ) are being developed for use in the treatment of heart failure. Accordingly, we compared the effects of amrinone on haemodynamic indices and myocardial metabolism with those of nitroprusside, a pure vasodilator. Previous within patient comparative studies of such agents useful in the treatment of cardiac failure are rare, ${ }^{10}$ and as far as we are aware all are concerned with simple haemodynamic measurements with no measurements of contractility or myocardial metabolism.

\section{Patients and methods}

Fourteen patients with impaired left ventricular function were studied. The aetiology of cardiac failure was dilated cardiomyopathy (two women, six men) and coronary artery disease* (one woman, five men). All were in sinus rhythm. The clinical details and current medication received by both groups of patients are

*The number of patients with coronary artery disease included in this study was fewer than we intended because the manufacturers of amrinone unilaterally withdrew all our stocks of the drug before completion of the trial. summarised in Table 1. Current medication was continued until the evening before the trial. The trial had the approval of the hospital ethical committee, and each patient gave informed written consent.

Studies were performed in the morning after an overnight fast. One hour before cardiac catheterisation the patients were given diazepam (10 $\mathrm{mg}$ ) and atropine $(0.6 \mathrm{mg})$ intramuscularly as premedication. Heparin (45 units $/ \mathrm{kg}$ ) was given intravenously at the same time to minimise the effect of a subsequent dose of heparin on free fatty acid concentrations. ${ }^{18}$ Routine right and left heart pressures were measured and cineangiography performed via catheters in the right femoral vein and artery inserted under local anaesthesia with lignocaine. Immediately after arterial catheterisation a second dose of heparin (45 units $/ \mathrm{kg}$ ) was given. Coronary arteriography was performed before the research procedure in four patients with dilated cardiomyopathy and in five with coronary artery disease. In the remaining five patients coronary arteriography was performed after the research procedure. The reason for this alteration in the protocol was that we were initially concerned about giving a possible positive inotropic agent (amrinone) to patients with unquantified coronary artery disease but subsequently tried to determine if the timing of angiography affected the results we obtained, which has been suggested by some workers. ${ }^{19}$ In each case the minimum volume of contrast was used (about five injections each of $5-8 \mathrm{ml}$ of $76 \%$ Urografin).

A catheter tip micromanometer (No 8 French gauge Gaeltec or Millar) was positioned in the left ventricle via the interatrial septum by the long sheath technique. ${ }^{20}$ Cardiac output was measured using indocyanine green dye injected into the left ventricle. 
The passage of green dye was detected using either a Nihon Kohden JQ-410V earpiece box and MLC-4100 cardiac output computer or a No 5 FG Schwarzer fibreoptic catheter connected to an IVH3 in vivo haemoreflector meter. ${ }^{6}$ Coronary sinus flow was measured by thermodilution as described previously. ${ }^{18}$ In 12 patients aortic pressure was measured using standard fluid filled catheters. Drugs were injected into a central vein. In seven patients a No 7 FG electromagnetic velocity probe (Carolina Medical Electronics) was inserted into the aortic root via an additional femoral artery puncture. This group of patients, which included all three with New York Heart Association grade II symptoms and four with grade III symptoms, were selected because they were considered clinically to have the least severe left ventricular impairment and to be most able to tolerate the additional catheterisation.

No measurements were made for at least 40 minutes after coronary angiography (when performed before the research procedure) or within 20 minutes of contrast injected elsewhere into the circulation. Pressures were measured with reference to the sternal angle as zero. Pressure, micromanometer, and Ganz thermistor signals were displayed on a Cambridge 12 channel recorder and stored on magnetic tape. Max $\mathrm{dP} / \mathrm{dt}, \min \mathrm{dP} / \mathrm{dt}$ (peak negative $\mathrm{dP} / \mathrm{dt}$ ), $\max \mathrm{dP} / \mathrm{dt} /$ $\mathrm{P},{ }^{21} \max (\mathrm{dP} / \mathrm{dt} / \mathrm{P}),{ }^{22} \mathrm{KVmax},{ }^{23}$ aortic flow, and indices derived from left ventricular pressure and aortic flow, including max $\mathrm{dPower} / \mathrm{dt},{ }^{24}$ were derived by online computer processing of the pressure and velocity signals by use of a Varian $620 / \mathrm{L}-100 .{ }^{6}$ (The velocity signal was converted to flow by multiplying it by the aortic cross sectional area determined by echocardiography.)

Left ventricular and coronary sinus blood were sampled. The oxygen content was measured with an Instrumentation Laboratories IL 282 gas analyser. Concentrations of lactate, pyruvate, hydroxybutyrate, acetoacetate, glycerol, and free fatty acid were determined as described. ${ }^{18}$ In four patients, two from each group, arterial glucose concentration was measured ${ }^{25}$ with a glucose analyser (Yellowsprings Instrument Company). Myocardial oxygen consumption, left ventricular minute work, myocardial efficiency, the extraction of substrates, extraction ratios, and oxygen extraction ratios of substrates were calculated using standard formulae. ${ }^{26}$

Measurements were made in the control state. Sodium nitroprusside was administered centrally at a rate of $55 \mu \mathrm{g} / \mathrm{min}$ using a constant infusion pump and, after allowing 15 minutes for stabilisation, measurements were repeated. Nine patients (four with coronary artery disease) also received an infusion at the rate of $110 \mu \mathrm{g} / \mathrm{min}$ with further measurements. The infusion was stopped, and after the return to the control state each patient received a bolus of $1.5 \mathrm{mg} /$ $\mathrm{kg}$ intravenous amrinone with further haemodynamic measurements seven minutes later. This interval was chosen because it has been used by others. ${ }^{3}$ Nine patients received a second dose of $2.0 \mathrm{mg} / \mathrm{kg}$ intravenous amrinone 15 minutes after the first dose, with further haemodynamic measurements seven minutes later. The patients who did not receive the high doses of the drugs were ones who had shown a pronounced reduction in left ventricular systolic or diastolic pressures with the lower dose of nitroprusside. Plasma amrinone concentrations were measured when the assay technique became available. Venous blood (11 samples) was taken at the time of haemodynamic measurements after amrinone administration in six patients (five of whom received two doses of the drug), and plasma concentrations of amrinone and its metabolite, $N$-acetyl amrinone, were subsequently measured by high pressure liquid chromatography. ${ }^{27}$

The batches of amrinone used in these experiments were DTD 223, DSF 175, and DUC 177 containing $10 \mathrm{mg} / \mathrm{ml}$ amrinone as the lactate. Each batch was bioassayed in our laboratory using normal female guinea pig papillary muscles to ascertain that an in vitro positive inotropic response was demonstrable. A solution of the carrier used in the amrinone ampoules was prepared according to the instructions supplied by Sterling-Winthrop Manufacturing Unit. It was identical in all respects to the amrinone solution except that it contained no amrinone. Its composition per $10 \mathrm{ml}$ ampoule was: lactic acid $90 \%$ w/w BP $0.112 \mathrm{ml}$, sodium metabisulphite $\mathrm{BP} 0.005 \mathrm{~g}, \mathrm{NaOH}$ $0.5 \mathrm{~mol}$ to adjust the $\mathrm{pH}$ to 3.5 , water for injection $\mathrm{BP}$ to $10 \mathrm{ml}$. Seven patients undergoing cardiac catheterisation received the carrier solution to determine its effect on acute haemodynamic indices and myocardial metabolism. The volume of carrier solution used in each case was $0.35 \mathrm{ml} / \mathrm{kg}$ being "equivalent" to $3.5 \mathrm{mg} / \mathrm{kg}$ amrinone solution.

\section{STATISTICAL ANALYSIS}

Values are expressed as means (standard error of the mean). Statistical analysis was by the paired $t$ test, $t$ test for small samples, and linear or log linear regression as appropriate. Where a relation was sought, but could not be detected using a curve fit, Spearman's rank test was used to test for a non-parametric relation. A p value of $<0.05$ was considered to be significant.

\section{Results}

COMPARISON OF CONTROL PERIODS IN BOTH GROUPS

The mean age of the patients with coronary artery 
Table 2 Effect of nitroprusside and amrinone on haemodynamics in patients with dilated cardiomyopathy.

Values are means (SEM)

\begin{tabular}{|c|c|c|c|c|c|c|c|}
\hline & \multirow[t]{2}{*}{$\boldsymbol{n}$} & \multirow[t]{2}{*}{ Control 1} & \multicolumn{2}{|l|}{ Nitroprusside } & \multirow[t]{2}{*}{ Control 2} & \multicolumn{2}{|l|}{ Amrinone } \\
\hline & & & $55 \mu \mathrm{g} / \mathrm{min}$ & $110 \mu \mathrm{g} / \mathrm{min}$ & & $1.5 \mathrm{mg} / \mathrm{kg}$ & $3.5 \mathrm{mg} / \mathrm{kg}$ \\
\hline Heart rate (beats/min) & $\left\{\begin{array}{l}8 \\
5\end{array}\right.$ & $\begin{array}{r}99 \cdot 0(7 \cdot 0) \\
108 \cdot 8(5 \cdot 2)\end{array}$ & $\begin{array}{r}95 \cdot 9(7 \cdot 2) \\
94 \cdot 8(6 \cdot 0)\end{array}$ & $\overline{1000(6.8)}$ & $97 \cdot 5(6 \cdot 5)$ & $99.0(6 \cdot 4)$ & - \\
\hline \multicolumn{8}{|l|}{ LV pressures $(\mathrm{mm} \mathrm{Hg})$ : } \\
\hline Peak systolic & $\left\{\begin{array}{l}8 \\
5\end{array}\right.$ & $\begin{array}{l}90 \cdot 3(5 \cdot 7) \\
94 \cdot 8(8 \cdot 1)\end{array}$ & $\begin{array}{l}79 \cdot 4(6 \cdot 1)^{\star \star} \\
84 \cdot 0(8 \cdot 9)^{\star \star}\end{array}$ & $\overline{80 \cdot 0(6 \cdot 7)^{\star \star}}$ & $\begin{array}{l}87 \cdot 3(4 \cdot 0) \\
85 \cdot 8(6 \cdot 2)\end{array}$ & $\begin{array}{l}83 \cdot 6(4 \cdot 1) \\
86 \cdot 2(6 \cdot 5)\end{array}$ & $\overline{82.8(6.9)}$ \\
\hline & & $21 \cdot 3(1 \cdot 8)$ & $10 \cdot 3(2 \cdot 0)^{\star \star}$ & - & $21 \cdot 4(1 \cdot 5)$ & $13 \cdot 1(2 \cdot 7)^{\star}$ & - \\
\hline Ena diastolic & & $24 \cdot 2(1 \cdot 8)$ & $12.0(2.9)^{\star \star}$ & $11 \cdot 6(2 \cdot 2)^{\star \star}$ & $22 \cdot 4(2 \cdot 0)$ & $16 \cdot 0(3 \cdot 1)^{\star}$ & $9 \cdot 6(3 \cdot 6)^{\star}$ \\
\hline Cardiac index $\left(1 / \mathrm{min} / \mathrm{m}^{2}\right)$ & & $1.60(0.21)$ & $2 \cdot 23(0 \cdot 29)^{\star \star}$ & - & $1.96(0.33)$ & $3.44(0.73)^{\star}$ & \\
\hline LV minute work & \}$_{8}^{3}$ & $\begin{array}{l}1.82(0.51) \\
2.51(0.51)\end{array}$ & $\begin{array}{l}2.44(0.43) \\
3.37(0.78)\end{array}$ & $-^{2.90(0.48)^{x}}$ & $\begin{array}{l}1.60(0.29) \\
2.90(0.62)\end{array}$ & $\begin{array}{l}3.04(0.64)^{\star} \\
5.11(1.14)^{\star \star}\end{array}$ & $-^{4 \cdot 40(1.43)}$ \\
\hline$(\mathbf{k g} \times \mathrm{m} / \mathrm{min})$ & 5 & $2.98(0.73)$ & $3.96(1.20)$ & $4 \cdot 36(1 \cdot 19)$ & $2 \cdot 30(0 \cdot 60)$ & $4.56(0.90)^{\star}$ & $7 \cdot 08(2 \cdot 70)$ \\
\hline Coronary sinus flow & $\left\{\begin{array}{l}8 \\
5\end{array}\right.$ & $161 \cdot 5(19 \cdot 6)$ & $133.3(12.9)$ & $\overline{140(190)}$ & $157 \cdot 9(20 \cdot 2)$ & $157 \cdot 8(17 \cdot 9)$ & \\
\hline$(\mathrm{ml} / \mathrm{min})$ & 5 & $179 \cdot 1(28 \cdot 2)$ & $142 \cdot 1(9 \cdot 8)$ & $146 \cdot 0(14 \cdot 0)$ & $163 \cdot 1(30 \cdot 9)$ & $155 \cdot 5(21 \cdot 8)$ & $158 \cdot 3(18 \cdot 7)$ \\
\hline $\begin{array}{l}\text { Aortocoronary sinus oxygen } \\
\text { difference }(\mathrm{ml} / 100 \mathrm{ml})\end{array}$ & $\left\{\begin{array}{l}8 \\
5\end{array}\right.$ & $\begin{array}{l}15 \cdot 1(0 \cdot 8) \\
13 \cdot 9(0 \cdot 5)\end{array}$ & $\begin{array}{l}14 \cdot 1(0 \cdot 8)^{\star \star} \\
12 \cdot 8(0 \cdot 5)^{\star}\end{array}$ & $\overline{11} \cdot 8(0.5)^{\star}$ & $\begin{array}{l}14 \cdot 7(0 \cdot 8) \\
14 \cdot 5(0 \cdot 9)\end{array}$ & $\begin{array}{l}13.6(0.9)^{\star} \\
13.0(0.7)^{\star \star}\end{array}$ & $\overline{12 \cdot 8(0 \cdot 6)^{\star}}$ \\
\hline Myocardial oxygen & 8 & $24 \cdot 5(2 \cdot 7)$ & $18 \cdot 8(1 \cdot 5)^{\star}$ & - & $23 \cdot 2(2 \cdot 8)$ & $21 \cdot 6(2 \cdot 3)$ & - \\
\hline consumption $(\mathrm{ml} / \mathrm{min})$ & 5 & $26 \cdot 0(4 \cdot 3)$ & $18 \cdot 8(1 \cdot 7)$ & $18 \cdot 2(2 \cdot 3)$ & $24 \cdot 2(4 \cdot 4)$ & $20 \cdot 8(4 \cdot 0)$ & $21 \cdot 0(2 \cdot 5)$ \\
\hline Efficiency $(\%)$ & $\left\{\begin{array}{l}8 \\
5\end{array}\right.$ & $\begin{array}{l}6 \cdot 0(0 \cdot 8) \\
6 \cdot 7(0.9)\end{array}$ & $\begin{array}{l}10 \cdot 7(1 \cdot 8)^{\star} \\
12 \cdot 0(2 \cdot 6)\end{array}$ & $\overline{1}_{14 \cdot 2(2 \cdot 6)^{\star}}$ & $\begin{array}{l}7 \cdot 7(1 \cdot 7) \\
5 \cdot 6(0 \cdot 6)\end{array}$ & $\begin{array}{l}15 \cdot 8(4 \cdot 5)^{\star} \\
13 \cdot 0(1 \cdot 7)^{\star \star}\end{array}$ & $\overline{18.8(5.9)}$ \\
\hline $\operatorname{Min} \mathrm{dP} / \mathrm{dt}\left(\mathrm{mm} \mathrm{Hg}^{\mathrm{mg}} \mathrm{s}\right)$ & 8 & $686 \cdot 1(57 \cdot 5)$ & $651.9(67 \cdot 0)^{\star}$ & - & $654 \cdot 5(42 \cdot 2)$ & $699 \cdot 8(69 \cdot 3)$ & - \\
\hline & & $739 \cdot 6(84 \cdot 4)$ & $713 \cdot 4(100 \cdot 5)$ & $672 \cdot 4(80 \cdot 3)^{\star}$ & $659 \cdot 0(62 \cdot 6)$ & $711 \cdot 0(105 \cdot 3)$ & $703 \cdot 0(65 \cdot 7)$ \\
\hline $\operatorname{Max} \mathrm{dP} / \mathrm{dt}(\mathrm{mm} \mathrm{Hg} / \mathrm{s})$ & $\left\{\begin{array}{l}8 \\
5\end{array}\right.$ & $\begin{array}{l}714 \cdot 8(26 \cdot 6) \\
729 \cdot 0(35 \cdot 5)\end{array}$ & $\begin{array}{l}696 \cdot 1(36 \cdot 0) \\
717 \cdot 6(57.9)\end{array}$ & $\overline{690} \cdot 0(50 \cdot 6)$ & $687.5(28.8)$ & $740 \cdot 3(56 \cdot 8)$ & $\overline{746.8(65.0)}$ \\
\hline $\mathrm{dP} / \mathrm{dt} /$ & & $25 \cdot 8(1.8)$ & $24 \cdot 5(1.8)$ & & $25 \cdot 5(1.3)$ & $\begin{array}{c}737 \cdot 8(77 \cdot 1) \\
25 \cdot 1(1 \cdot 8)\end{array}$ & $746.8(65.9)$ \\
\hline $\operatorname{Nadx}$ ar $/ \mathrm{det} / \mathrm{T} / \mathrm{s}$ & & $24 \cdot 6(1 \cdot 4)$ & $22.4(1.4)^{\star}$ & $23 \cdot 4(1.4)$ & $24 \cdot 8(1 \cdot 2)$ & $25 \cdot 6(2 \cdot 7)$ & $24 \cdot 8(1 \cdot 0)$ \\
\hline $\operatorname{Max}(d \mathbf{P} / \mathrm{dt} / \mathrm{P}) / \mathrm{s}$ & $\{8$ & $103 \cdot 5(3.3)$ & $106 \cdot 3(6 \cdot 0)$ & - & $105 \cdot 1(5 \cdot 4)$ & $104 \cdot 8(4 \cdot 6)$ & \\
\hline & & $106 \cdot 8(4 \cdot 0)$ & $109 \cdot 8(7 \cdot 2)$ & $110 \cdot 6(5 \cdot 7)$ & $111 \cdot 8(6 \cdot 7)$ & $110 \cdot 8(5 \cdot 5)$ & $112 \cdot 2(3 \cdot 8)$ \\
\hline $\mathrm{KV} \max / \mathrm{s}$ & $\left\{\begin{array}{l}8 \\
5\end{array}\right.$ & $76 \cdot 9(4 \cdot 0)$ & $73 \cdot 3(3 \cdot 8)$ & $\overline{7}_{72,0(5,4)}$ & $75 \cdot 9(3 \cdot 5)$ & $76 \cdot 1(5 \cdot 1)$ & $\overline{78}$ \\
\hline & & $77 \cdot 4(4 \cdot 7)$ & $72 \cdot 0(4 \cdot 7)$ & $73 \cdot 8(5 \cdot 4)$ & $77 \cdot 8(4 \cdot 7)$ & $80 \cdot 8(5 \cdot 0)$ & $78 \cdot 0(2 \cdot 8)$ \\
\hline
\end{tabular}

${ }^{\star} \mathrm{p}<0.05{ }^{\star \star} \mathrm{p}<0.01 \quad$ (significance of difference from control: nitroprusside $v$ control 1, amrinone $v$ control 2).

Table 3 Effect of nitroprusside and amrinone on haemodynamics in patients with coronary artery disease.

Values are means (SEM)

\begin{tabular}{|c|c|c|c|c|c|c|c|}
\hline & \multirow[t]{2}{*}{$n$} & \multirow[t]{2}{*}{ Control 1} & \multicolumn{2}{|l|}{ Nitroprusside } & \multirow[t]{2}{*}{ Control 2} & \multicolumn{2}{|l|}{ Amrinone } \\
\hline & & & $55 \mu \mathrm{g} / \mathrm{min}$ & $110 \mu \mathrm{g} / \min$ & & $1.5 \mathrm{mg} / \mathrm{kg}$ & $3.5 \mathrm{mg} / \mathrm{kg}$ \\
\hline Heart rate (beats/min) & $\left\{\begin{array}{l}6 \\
4\end{array}\right.$ & $\begin{array}{l}93 \cdot 8(6 \cdot 7) \\
98 \cdot 0(9 \cdot 6)\end{array}$ & $93 \cdot 0(7 \cdot 3)$ & $\overline{0}_{07.8(13.0)}$ & $89 \cdot 7(6 \cdot 6)$ & $94 \cdot 5(8 \cdot 7)$ & $\overline{100.5(13.7)}$ \\
\hline \multicolumn{8}{|l|}{ LV pressures $(\mathrm{mm} \mathrm{Hg})$ : } \\
\hline Peak systolic & $\left\{\begin{array}{l}6 \\
4\end{array}\right.$ & $\begin{array}{l}94 \cdot 7(5 \cdot 1) \\
94 \cdot 5(7 \cdot 5)\end{array}$ & $\begin{array}{l}87 \cdot 0(5 \cdot 5) \\
86 \cdot 0(7 \cdot 9)^{\star \star}\end{array}$ & $\overline{82.0(6.5)^{\star \star}}$ & $\begin{array}{l}94 \cdot 7(5 \cdot 3) \\
96 \cdot 5(8 \cdot 2)\end{array}$ & $\begin{array}{l}91 \cdot 3(5 \cdot 8) \\
93 \cdot 0(6 \cdot 1)\end{array}$ & $\overline{87 \cdot 3(5 \cdot 8)^{\star}}$ \\
\hline End diastolic & & $21 \cdot 3(2 \cdot 5)$ & $13 \cdot 8(3 \cdot 1)^{\star \star}$ & - & $19 \cdot 8(3 \cdot 3)$ & $15 \cdot 7(4 \cdot 4)$ & - \\
\hline End diastonic & & $23 \cdot 3(3 \cdot 4)$ & $16 \cdot 0(4 \cdot 0)^{\star \star}$ & $14 \cdot 0(5 \cdot 2)^{\star}$ & $22 \cdot 3(4 \cdot 4)$ & $18 \cdot 8(6 \cdot 2)$ & $14 \cdot 8(5 \cdot 9)^{\star}$ \\
\hline Cardiac index $\left(1 / \mathrm{min} / \mathrm{m}^{2}\right)$ & $\{6$ & $\begin{array}{l}1.87(0 \cdot 25) \\
1.88(0.37)\end{array}$ & $\begin{array}{l}2.37(0.54) \\
2.03(0.42)\end{array}$ & -2.4000 .26 & $2 \cdot 13(0 \cdot 43)$ & $2 \cdot 85(0 \cdot 46)^{\star}$ & - \\
\hline LV minute work & $\{6$ & $3.57(0.78)$ & $4.40(1.27)$ & $-2.40(0.20)$ & $\begin{array}{l}1.90(0.53) \\
4 \cdot 15(1.06)\end{array}$ & $\begin{array}{l}2.85(0.40)^{\star} \\
5.58(1.24)^{\star}\end{array}$ & $-^{4.03(0.93)}$ \\
\hline$(\mathrm{kg} \times \mathrm{m} / \mathrm{min})$ & & $3.62(1.23)$ & $3.74(1.50)$ & $4 \cdot 36(1 \cdot 39)$ & $3.95(1.52)$ & $5.39(1.48)^{\star}$ & $7.01(1.58)$ \\
\hline Coronary sinus flow & 6 & $128 \cdot 0(13.4)$ & $108 \cdot 8(11 \cdot 9)^{\star}$ & & $135 \cdot 0(20 \cdot 4)$ & $127 \cdot 0(12 \cdot 2)$ & \\
\hline$(\mathrm{ml} / \mathrm{min})$ & 4 & $129 \cdot 2(20 \cdot 6)$ & $113 \cdot 5(18 \cdot 2)$ & $133 \cdot 5(25 \cdot 2)$ & $134 \cdot 2(30 \cdot 4)$ & $135 \cdot 9(16 \cdot 5)$ & $134 \cdot 1(10 \cdot 4)$ \\
\hline Aortocoronary sinus oxygen & 6 & $12.5(0 \cdot 8)$ & $12 \cdot 1(0 \cdot 8)$ & - & $12 \cdot 1(0 \cdot 8)$ & $11 \cdot 7(0.9)$ & - \\
\hline difference $(\mathrm{ml} / 100 \mathrm{ml})$ & 4 & $12 \cdot 2(0 \cdot 7)$ & $12 \cdot 2(0 \cdot 6)$ & $11 \cdot 7(0 \cdot 8)$ & $12 \cdot 1(0 \cdot 7)$ & $11 \cdot 4(0.8)$ & $11 \cdot 1(1 \cdot 0)$ \\
\hline Myocardial oxygen & $\{6$ & $16 \cdot 4(2 \cdot 4)$ & $13 \cdot 3(2 \cdot 0)^{\star}$ & - & $16.9(3.4)$ & $14.9(1.9)$ & - \\
\hline consumption $(\mathrm{ml} / \mathrm{min})$ & 4 & $16 \cdot 1(3 \cdot 4)$ & $14 \cdot 1(2 \cdot 9)$ & $16 \cdot 2(4 \cdot 2)$ & $16 \cdot 7(4 \cdot 7)$ & $15 \cdot 7(2 \cdot 9)$ & $15 \cdot 1(2 \cdot 1)$ \\
\hline Efficiency $(\%)$ & $\left\{\begin{array}{l}6 \\
4\end{array}\right.$ & $\begin{array}{l}13 \cdot 2(1 \cdot 7) \\
13 \cdot 2(2 \cdot 3)\end{array}$ & $\begin{array}{l}18 \cdot 8(3.9) \\
14.5(2.6)\end{array}$ & $\overline{15} \cdot 6(1.5)$ & $\begin{array}{l}14 \cdot 2(1 \cdot 3) \\
13 \cdot 3(1 \cdot 7)\end{array}$ & $\begin{array}{l}21 \cdot 8(3 \cdot 4) \\
19 \cdot 9(2 \cdot 6)^{\star \star}\end{array}$ & $\overline{2}^{27 \cdot 3(5 \cdot 2) \star}$ \\
\hline $\operatorname{Min~} \mathrm{dP} / \mathrm{dt}(\mathrm{mm} \mathrm{Hg} / \mathrm{s})$ & $\{6$ & $717 \cdot 2(63 \cdot 4)$ & $656 \cdot 0(56 \cdot 0)$ & - & $711 \cdot 7(67 \cdot 1)$ & $721 \cdot 0(68 \cdot 3)$ & \\
\hline & & $682 \cdot 8(79 \cdot 3)$ & $602 \cdot 8(70 \cdot 3)$ & $606 \cdot 5(67 \cdot 5)$ & $683.5(91.5)$ & $730 \cdot 0(101 \cdot 0)$ & $697 \cdot 3(75 \cdot 0)$ \\
\hline $\operatorname{Max} \mathrm{dP} / \mathrm{dt}\left(\mathrm{mm} \mathrm{Hg}_{\mathrm{g}} / \mathrm{s}\right)$ & $\{6$ & $\begin{array}{l}771 \cdot 8(80 \cdot 4) \\
752 \cdot 0(109 \cdot 6)\end{array}$ & $\begin{array}{l}708 \cdot 0(57 \cdot 3) \\
678 \cdot 5(81 \cdot 4)\end{array}$ & $\overline{676} \cdot 0(79 \cdot 9)$ & $\begin{array}{l}769 \cdot 7(80 \cdot 3) \\
766 \cdot 5(122 \cdot 3)\end{array}$ & $\begin{array}{l}810 \cdot 0(76 \cdot 7) \\
824 \cdot 5(118 \cdot 1)^{\star}\end{array}$ & $\overline{798} \cdot 3(87 \cdot 1)$ \\
\hline $\operatorname{Max} \mathrm{dP} / \mathrm{dt} / \mathrm{P}\left(\mathrm{s}^{-1}\right)$ & 6 & $\begin{array}{l}24 \cdot 3(2 \cdot 1) \\
25 \cdot 0(2 \cdot 5)\end{array}$ & $\begin{array}{l}25 \cdot 3(2 \cdot 7) \\
27 \cdot 0(3 \cdot 8)\end{array}$ & $\overline{2}^{26.5(3.8)}$ & $\begin{array}{l}24 \cdot 7(2 \cdot 1) \\
25 \cdot 8(3 \cdot 2)\end{array}$ & $\begin{array}{l}26 \cdot 5(1.4) \\
27.3(2.0)\end{array}$ & 26.0(3.9) \\
\hline & $\{6$ & $98 \cdot 7(3 \cdot 4)$ & $99 \cdot 5(4 \cdot 1)$ & - & $101 \cdot 7(4 \cdot 6)$ & $103 \cdot 3(5 \cdot 5)$ & \\
\hline $\operatorname{Max}(\mathrm{dP} / \mathrm{dt} / \mathrm{P})\left(\mathrm{s}^{-1}\right)$ & 4 & $102 \cdot 0(4 \cdot 1)$ & $103 \cdot 8(4 \cdot 1)$ & $100 \cdot 8(7 \cdot 2)$ & $103 \cdot 3(6 \cdot 7)$ & $108 \cdot 8(5 \cdot 2)$ & $106 \cdot 8(5 \cdot 3)$ \\
\hline $\mathrm{KV} \max \left(\mathrm{s}^{-1}\right)$ & $\left\{\begin{array}{l}6 \\
4\end{array}\right.$ & $\begin{array}{l}72 \cdot 7(6 \cdot 4) \\
77 \cdot 0(8 \cdot 6)\end{array}$ & $\begin{array}{l}75 \cdot 7(7 \cdot 4) \\
81 \cdot 5(10 \cdot 1)\end{array}$ & $\overline{76} \cdot 3(8.9)$ & $\begin{array}{l}69 \cdot 5(4 \cdot 6) \\
71 \cdot 0(7 \cdot 0)\end{array}$ & $\begin{array}{l}79 \cdot 0(5 \cdot 5)^{\star} \\
82 \cdot 3(8 \cdot 0)\end{array}$ & $\overline{80} \cdot 8(9 \cdot 2)$ \\
\hline
\end{tabular}

${ }^{\star} \mathrm{p}<0.05 \quad{ }^{\star \star} \mathrm{p}<0.01 \quad$ (significance of difference from control: nitroprusside $v$ control 1 and amrinone $v$ control 2). 
disease was higher $(p<0.05)$ than that of those with dilated cardiomyopathy (Table 1). The length of history of cardiac failure and angiographically derived left ventricular volume data were not significantly different in the two groups. In the control state the resting haemodynamic values in the patients with dilated cardiomyopathy (Table 2) were similar to those in the patients with coronary artery disease (Table 3 ) except that the patients with coronary artery disease had a lower arteriocoronary sinus oxygen difference $(12.5 \mathrm{v}$ $15.1 \mathrm{ml} / 100 \mathrm{ml}, \mathrm{p}<0.05)$ and a higher efficiency $(13.2$ $v 6.0 \%, p<0.01$ ). The difference between the control myocardial oxygen consumptions in the two groups just failed to achieve statistical significance $(0.05<\mathrm{p}<0.1)$.

In the control state there was no difference between metabolism of lactate, pyruvate, hydroxybutyrate, acetoacetate, glycerol, or free fatty acid in the two groups as assessed by the arterial and coronary sinus concentrations, myocardial extractions, extraction ratios, or oxygen extraction ratios of the substrates (Tables 4 and 5).

\section{COMPARISON OF CONTROL PERIODS WITHIN EACH GROUP}

In each group the haemodynamic (Tables 2 and 3 ) and metabolic (Tables 4 and 5) measurements during the first control period (before nitroprusside) were the same as those during the second control period (before amrinone), confirming a complete return to the baseline status after discontinuation of the nitroprusside infusion.

\section{EFFECTS ON HAEMODYNAMICS}

In the patients with dilated cardiomyopathy heart rate tended to fall with nitroprusside and to rise with amrinone, but the changes were not statistically significant (Table 2). Left ventricular peak systolic pressure and myocardial oxygen consumption fell in a dose related manner with nitroprusside. Smaller changes with amrinone did not achieve statistical significance. Cardiac index and efficiency rose and left ventricular end diastolic pressure fell in dose related manners with both drugs. Left ventricular minute work tended to rise with both drugs but was significant for only the low dose of amrinone. Coronary sinus flow tended to fall with nitroprusside (not significant) but was unaltered by amrinone. Min $\mathrm{dP} / \mathrm{dt}$ was significantly reduced by nitroprusside but not by amrinone. Max dP/dt tended to fall with nitroprusside and to rise with amrinone, but the changes were small and not significant. Other pressure derived indices of left ventricular contractility were unaltered by either drug.

In the patients with coronary artery disease changes in haemodynamic indices similar to those in patients with dilated cardiomyopathy occurred during administration of the two drugs (Table 3), although statistically these changes were less pronounced, possibly because of the smaller number of patients in this group.

In both groups combined, $\max \mathrm{dP} / \mathrm{dt}$ increased after the highest dose of both nitroprusside and amrinone in four patients and decreased after both drugs in five patients. In the remaining five patients $\max \mathrm{dP} / \mathrm{dt}$ fell with nitroprusside but rose after amrinone, but in this subgroup the drugs had opposite effects on heart rate; heart rate decreased after nitroprusside in all five patients but was either unchanged $(n=2)$ or increased $(n=3)$ after amrinone. In this subgroup of five patients the two drugs had opposite effects on heart rate, which were both statistically $(\mathrm{p}<0.02)$ and clinically different $(10$ (2) beats/minute).

\section{EFFECTS ON METABOLISM}

Arterial lactate concentration tended to fall with nitroprusside but not with amrinone in both groups (Tables 4 and 5). The myocardial extraction ratios of lactate tended to fall in both groups during administration of each drug, although the changes were not statistically significant. In five patients with dilated cardiomyopathy and five with coronary artery disease lactate extraction was normal $(>10 \%)$ in the control state. In three of the patients with dilated cardiomyopathy the lactate extraction remained normal after administration of both drugs. Lactate extraction in one patient with coronary artery disease and one with cardiomyopathy became abnormal $(<10 \%)$ after nitroprusside but not amrinone. In the remaining five patients the extraction ratio became abnormal only after amrinone.

Four patients, three of whom had dilated cardiomyopathy, had abnormal lactate extraction $(<10 \%)$ in the control state. Both drugs produced no alteration in lactate extraction in the three patients with dilated cardiomyopathy but an improvement in the one patient with coronary artery disease. Arterial and coronary sinus concentrations, extraction ratios, and oxygen extraction ratios of pyruvate, hydroxybutyrate, and acetoacetate were unaffected by either drug in either group (Tables 4 and 5)

Arterial concentrations of free fatty acid tended to rise in each group with both drugs, although the changes were most apparent after amrinone (Tables 4 and 5). Furthermore, these changes after amrinone were accompanied by increases in arterial and coronary sinus glycerol concentrations and an increase in coronary sinus free fatty acid concentration.

In the four patients in whom measurements were made, nitroprusside did not alter arterial glucose concentration, whereas intravenous amrinone increased 
Table 4 Effect of nitroprusside and amrinone on myocardial metabolism in patients with dilated cardiomyopathy. Values are means (SEM)

\begin{tabular}{|c|c|c|c|c|c|c|c|}
\hline & \multirow[t]{2}{*}{$n$} & \multirow[t]{2}{*}{ Control 1} & \multicolumn{2}{|l|}{ Nitroprusside } & \multirow[t]{2}{*}{ Control 2} & \multicolumn{2}{|l|}{ Amrinone } \\
\hline & & & $55 \mu \mathrm{g} / \min$ & $110 \mu \mathrm{g} / \mathrm{min}$ & & $1.5 \mathrm{mg} / \mathrm{kg}$ & $3.5 \mathrm{mg} / \mathrm{kg}$ \\
\hline \multicolumn{8}{|l|}{ Lactate } \\
\hline $\begin{array}{l}\text { A } \\
\text { CS } \\
\text { ER } \\
\text { OER }\end{array}$ & $\left\{\begin{array}{l}8 \\
5 \\
8 \\
5 \\
8 \\
5 \\
8 \\
5\end{array}\right.$ & $\begin{array}{c}0.863(0.076) \\
0.941(0 \cdot 103) \\
0.634(0 \cdot 111) \\
0.724(0 \cdot 165) \\
29 \cdot 5(8 \cdot 0) \\
27 \cdot 7(11 \cdot 2) \\
11 \cdot 4(2 \cdot 7) \\
11 \cdot 6(3 \cdot 7)\end{array}$ & $\begin{array}{c}0.775(0.085) \star \\
0.828(0.133) \\
0.605(0.077) \\
0.663(0 \cdot 113) \\
20 \cdot 3(6.9) \\
18 \cdot 1(8.5) \\
8 \cdot 8(3.3) \\
9 \cdot 3(4.8)\end{array}$ & $\begin{array}{l}\overline{0} \cdot 821(0 \cdot 136) \\
\overline{0} \cdot 634(0 \cdot 121) \\
\overline{21} \cdot 1(10 \cdot 3) \\
\overline{12} \cdot 1(5 \cdot 2)\end{array}$ & $\begin{array}{c}0.882(0.075) \\
0.959(0.085) \\
0.683(0.076) \\
0.765(0.088) \\
22.5(6.2) \\
19 \cdot 8(7.0) \\
10.5(2.7) \\
9.8(3.3)\end{array}$ & $\begin{array}{l}0.815(0.070) \\
0.854(0.089) \\
0.630(0.071) \\
0 \cdot 632(0.114) \\
21 \cdot 5(6.4) \\
25 \cdot 6(9.4) \\
10 \cdot 1(3.4) \\
12 \cdot 2(4.9)\end{array}$ & $\begin{array}{l}-0.799(0.107) \\
-\quad 0.690(0.127) \\
-\quad 17 \cdot 4(7 \cdot 7) \\
-6.8(2 \cdot 8)\end{array}$ \\
\hline \multicolumn{8}{|c|}{ Pyruvate } \\
\hline $\begin{array}{l}\text { CS } \\
\text { ER } \\
\text { OER }\end{array}$ & $\left\{\begin{array}{l}8 \\
5 \\
8 \\
5 \\
8 \\
5 \\
8 \\
5\end{array}\right.$ & $\begin{array}{c}0.057(0.007) \\
0.064(0.010) \\
0.044(0.004) \\
0.049(0.003) \\
17.6(8.6) \\
17 \cdot 4(11 \cdot 1) \\
0.6(0.2) \\
0.7(0.4)\end{array}$ & $\begin{array}{c}0.054(0.006) \\
0.060(0.008) \\
0.045(0.004) \\
0.050(0.004) \\
8 \cdot 5(11 \cdot 3) \\
12 \cdot 7(11.4) \\
0.5(0.2) \\
0.6(0.3)\end{array}$ & $\begin{array}{l}\overline{0} .065(0.010) \\
\overline{0} \cdot 048(0.002) \\
\frac{17}{17} \cdot 2(14.5) \\
\overline{1} \cdot 0(0.4)\end{array}$ & $\begin{array}{c}0.061(0.006) \\
0.065(0.009) \\
0.047(0.004) \\
0.048(0.006) \\
17 \cdot 7(10 \cdot 4) \\
21.7(14.2) \\
0.7(0.3) \\
0.8(0.3)\end{array}$ & $\begin{array}{c}0.062(0.007) \\
0.069(0.010) \\
0.045(0.003) \\
0.049(0.004) \\
23.1(6.4) \\
25.0(10.1) \\
0.8(0.3) \\
0.9(0.4)\end{array}$ & $\begin{array}{l}-0.068(0.007) \\
-\quad 0.049(0.006) \\
-\quad 27 \cdot 7(8.0) \\
-0.9(0.3)\end{array}$ \\
\hline \multicolumn{8}{|c|}{ Hydroxybutyrate } \\
\hline $\begin{array}{l}\text { A } \\
\text { CS } \\
\text { ER } \\
\text { OER }\end{array}$ & $\left\{\begin{array}{l}8 \\
5 \\
8 \\
5 \\
8 \\
5 \\
8 \\
5\end{array}\right.$ & $\begin{array}{c}0 \cdot 358(0 \cdot 117) \\
0 \cdot 323(0 \cdot 113) \\
0 \cdot 210(0 \cdot 068) \\
0 \cdot 195(0 \cdot 068) \\
37 \cdot 6(5 \cdot 5) \\
32 \cdot 2(7 \cdot 8) \\
10 \cdot 9(3 \cdot 4) \\
10 \cdot 6(4 \cdot 2)\end{array}$ & $\begin{array}{c}0 \cdot 344(0 \cdot 103) \\
0 \cdot 303(0 \cdot 109) \\
0 \cdot 211(0 \cdot 061) \\
0 \cdot 189(0 \cdot 064) \\
34 \cdot 7(4 \cdot 5) \\
30 \cdot 6(6 \cdot 6) \\
10 \cdot 6(3 \cdot 1) \\
10 \cdot 1(4 \cdot 1)\end{array}$ & $\begin{array}{l}\overline{0} \cdot 256(0.092) \\
\frac{0}{0} \cdot 179(0.070) \\
\frac{33}{3} \cdot 1(4 \cdot 1) \\
7 \cdot 3(2 \cdot 2)\end{array}$ & $\begin{array}{c}0.278(0.097) \\
0.230(0.094) \\
0 \cdot 172(0.058) \\
0 \cdot 133(0.047) \\
33.9(3.9) \\
35.9(5.6) \\
8 \cdot 2(3.2) \\
8 \cdot 4(4.5)\end{array}$ & $\begin{array}{c}0.328(0.098) \\
0.215(0.070) \\
0.194(0.059) \\
0.120(0.040) \\
34.9(8.2) \\
34.0(13.6) \\
10.9(3.0) \\
9 \cdot 1(4.3)\end{array}$ & $\begin{array}{l}-0.263(0.095) \\
-\quad 0.185(0.063) \\
-24 \cdot 1(7 \cdot 1) \\
-7 \cdot 6(3.2)\end{array}$ \\
\hline \multicolumn{6}{|c|}{ Acetoacetate } & & \\
\hline $\begin{array}{l}\text { A } \\
\text { CS } \\
\text { ER } \\
\text { OER }\end{array}$ & $\left\{\begin{array}{l}8 \\
5 \\
8 \\
5 \\
8 \\
5 \\
8 \\
5\end{array}\right.$ & $\begin{array}{c}0 \cdot 177(0.046) \\
0 \cdot 146(0.033) \\
0 \cdot 101(0.025) \\
0.090(0.020) \\
39 \cdot 7(5 \cdot 0) \\
36 \cdot 6(7.1) \\
4.9(1.3) \\
4 \cdot 1(1.4)\end{array}$ & $\begin{array}{c}0.167(0.043) \\
0.136(0.032) \\
0.095(0.022) \\
0.084(0.019) \\
39 \cdot 0(5 \cdot 1) \\
35 \cdot 6(7 \cdot 3) \\
5 \cdot 0(1.4) \\
4 \cdot 2(1.4)\end{array}$ & $\begin{array}{l}\overline{0} \cdot 119(0.026) \\
\overline{0} \cdot 076(0.016) \\
\overline{34} \cdot 4(5 \cdot 2) \\
\overline{3} \cdot 6(1 \cdot 0)\end{array}$ & $\begin{array}{c}0 \cdot 153(0 \cdot 041) \\
0 \cdot 118(0.026) \\
0 \cdot 090(0.023) \\
0 \cdot 076(0 \cdot 018) \\
38 \cdot 0(4 \cdot 7) \\
33 \cdot 5(6 \cdot 8) \\
4 \cdot 1(1 \cdot 2) \\
3 \cdot 0(1 \cdot 2)\end{array}$ & $\begin{array}{c}0.166(0.044) \\
0 \cdot 109(0.023) \\
0.092(0.020) \\
0.071(0.011) \\
37 \cdot 7(5 \cdot 9) \\
30 \cdot 2(7 \cdot 7) \\
5 \cdot 1(1 \cdot 3) \\
3 \cdot 1(1 \cdot 2)\end{array}$ & $\begin{array}{l}-0.118(0.026) \\
-\quad 0.072(0.016) \\
-\quad 37 \cdot 7(7.6) \\
-3.8(1.2)\end{array}$ \\
\hline \multicolumn{8}{|l|}{ Glycerol } \\
\hline $\begin{array}{l}\text { A } \\
\text { CS } \\
\text { ER } \\
\text { OER }\end{array}$ & $\left\{\begin{array}{l}8 \\
5 \\
8 \\
5 \\
8 \\
5 \\
8 \\
5\end{array}\right.$ & $\begin{array}{c}0 \cdot 121(0 \cdot 019) \\
0 \cdot 124(0 \cdot 028) \\
0 \cdot 105(0 \cdot 019) \\
0 \cdot 017(0 \cdot 028) \\
14 \cdot 3(4 \cdot 6) \\
14 \cdot 0(7 \cdot 2) \\
1 \cdot 0(0 \cdot 4) \\
1 \cdot 1(0 \cdot 7)\end{array}$ & $\begin{array}{c}0.102(0.018) \\
0.099(0.026) \\
0.097(0.017) \\
0.094(0.022) \\
1.0(6.5) \\
-1.6(10.4) \\
0.4(0 \cdot 2) \\
0.5(0.3)\end{array}$ & $\begin{array}{l}\overline{0} \cdot 108(0.017) \\
-0 \cdot 072(0.022) \\
-30 \cdot 8(17 \cdot 1) \\
-2 \cdot 7(1 \cdot 5)\end{array}$ & $\begin{array}{c}0.093(0.015) \\
0.087(0.023) \\
0.083(0.014) \\
0.078(0.021) \\
10.7(5.0) \\
9.0(6.9) \\
0.6(0.2) \\
0.5(0.3)\end{array}$ & $\begin{array}{l}0 \cdot 145(0.020)^{\star} \\
0 \cdot 120(0.024) \\
0 \cdot 128(0.019)^{\star} \\
0 \cdot 113(0.028) \\
12 \cdot 0(3.8) \\
8 \cdot 2(5.4) \\
1 \cdot 2(0.3) \\
0.7(0.3)\end{array}$ & $\begin{array}{l}-0.123(0.028) \\
-0.128(0.025) \\
-\quad 8.3(11.2) \\
-0.5(0.4)\end{array}$ \\
\hline \multicolumn{8}{|c|}{ Free fatty acid } \\
\hline $\begin{array}{l}\text { A } \\
\text { CS } \\
\text { ER }\end{array}$ & $\left\{\begin{array}{l}8 \\
5 \\
8 \\
5 \\
8 \\
5 \\
8 \\
5\end{array}\right.$ & $\begin{array}{c}0 \cdot 89(0 \cdot 12) \\
0 \cdot 90(0 \cdot 16) \\
0 \cdot 68(0 \cdot 12) \\
0 \cdot 74(0 \cdot 15) \\
26 \cdot 3(6 \cdot 2) \\
19 \cdot 2(4 \cdot 2) \\
78 \cdot 2(12 \cdot 4) \\
66 \cdot 8(14 \cdot 4)\end{array}$ & $\begin{array}{c}0.94(0.09) \\
0.94(0.09) \\
0 \cdot 72(0 \cdot 12) \\
0 \cdot 80(0 \cdot 14) \\
26 \cdot 2(8 \cdot 4) \\
16 \cdot 4(9 \cdot 3) \\
95 \cdot 4(17 \cdot 0) \\
78 \cdot 2(25 \cdot 0)\end{array}$ & $\begin{array}{l}\overline{0} \cdot 89(0 \cdot 17) \\
\overline{0} \cdot 70(0 \cdot 18) \\
\overline{23} \cdot 1(8 \cdot 9) \\
\overline{90} \cdot 4(29 \cdot 4)\end{array}$ & $\begin{array}{c}0.77(0.09) \\
0.72(0.09) \\
0.57(0 \cdot 10) \\
0.50(0.13) \\
27 \cdot 8(6 \cdot 6) \\
34 \cdot 5(8 \cdot 5) \\
76 \cdot 4(14 \cdot 7) \\
88 \cdot 4(16.5)\end{array}$ & $\begin{array}{l}0.98(0.09) \\
0.82(0.08) \\
0.83(0.11)^{\star} \\
0.64(0.09) \\
18.0(4.4)^{\star} \\
23.3(5.2) \\
64.4(12.1) \\
79.8(11.9)\end{array}$ & $\begin{array}{l}-0.98(0.15) \\
-0.75(0.16) \\
{ }^{2} 4.3(10.1) \\
-109.4(38.9)\end{array}$ \\
\hline
\end{tabular}

A, arterial concentration (mmol); CS, coronary sinus concentration (mmol); ER, extraction ratio (\%); OER, oxygen extraction ratio (\%). ${ }^{\star} \mathrm{p}<0.05$ (significant difference from control: nitroprusside $v$ control 1 and amrinone $v$ control 2 ).

the glucose concentration in each patient (Fig. 1). The increases in glucose concentration after amrinone were clinically significant in three of the four.

EFFECTS OF TIMING OF CORONARY ANGIOGRAPHY The initial control haemodynamic and metabolic measurements and the subsequent effects of drug interventions were not affected by whether coronary arteriography was performed before $(n=9)$ or after $(n=5)$ the drug interventions. Max dP/dt fell with successive doses of amrinone in three of the five patients who did not have coronary arteriography until after the drug study, whereas it rose on successive doses in the remaining two patients. Interestingly, in each of these five patients the low and high doses of nitroprusside produced changes in $\max \mathrm{dP} /$ 
Table 5 Effect of nitroprusside and amrinone on myocardial metabolism in patients with coronary artery disease.

Values are means (SEM)

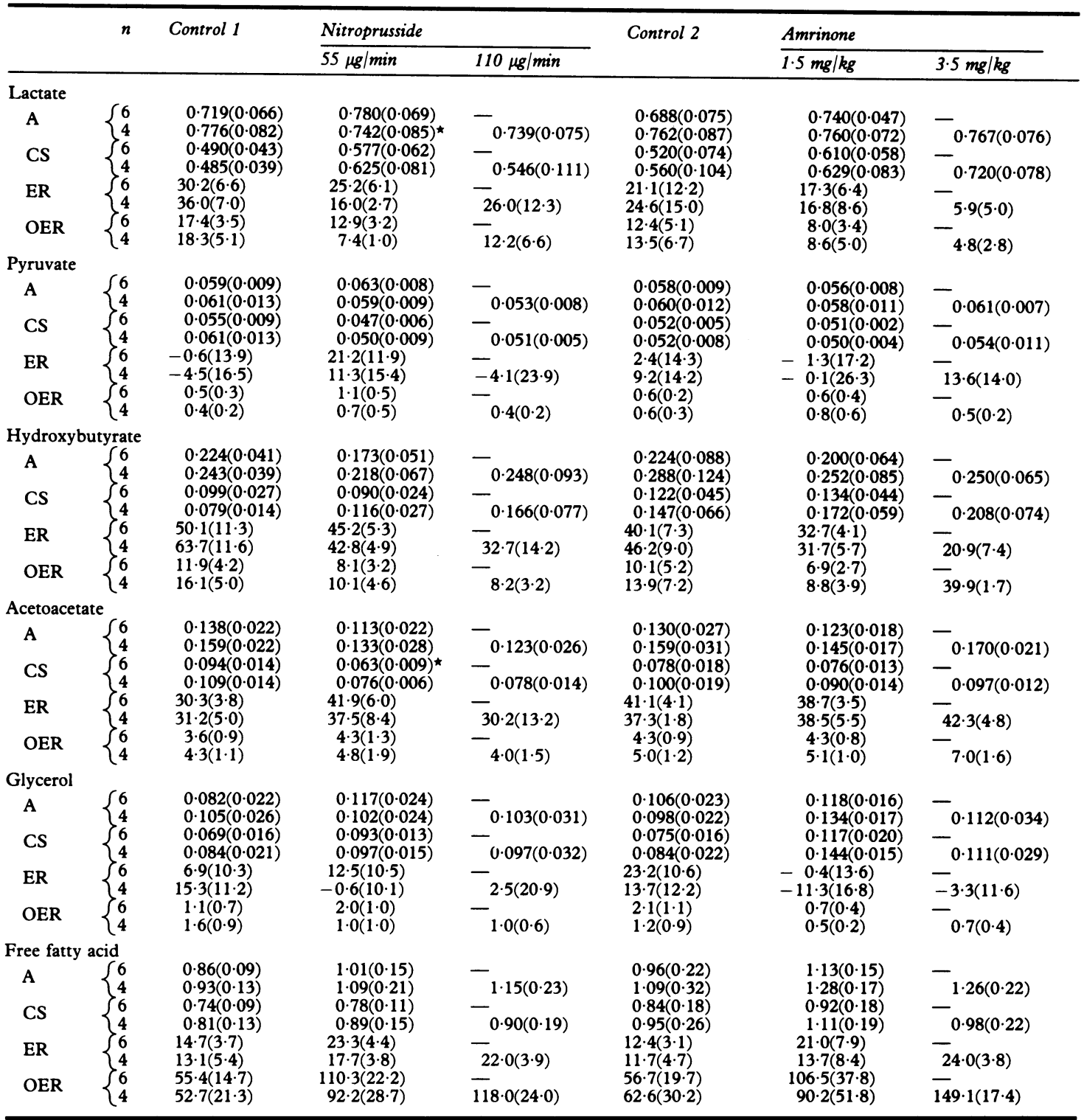

A, arterial concentration (mmol); CS, coronary sinus concentration (mmol); ER, extraction ratio (\%); OER, oxygen extraction ratio (\%). ${ }^{\star} \mathrm{p}<0.05$ (significant difference from control: nitroprusside $v$ control 1 and amrinone $v$ control 2).

$\mathrm{dt}$, which were in the same direction as the changes in $\max \mathrm{dP} / \mathrm{dt}$ produced by corresponding low or high doses of amrinone.

COMPARISON OF THE EFFECTS OF AMRINONE AND NITROPRUSSIDE

As a general rule it was noticeable that in most of the
14 patients studied, the haemodynamic effects of nitroprusside mimicked the changes in the same indices after amrinone in that individual, except for the changes in heart rate and max $\mathrm{dP} / \mathrm{dt}$ noted above. For the groups as a whole the response to nitroprusside was not significantly different from the response to amrinone. 

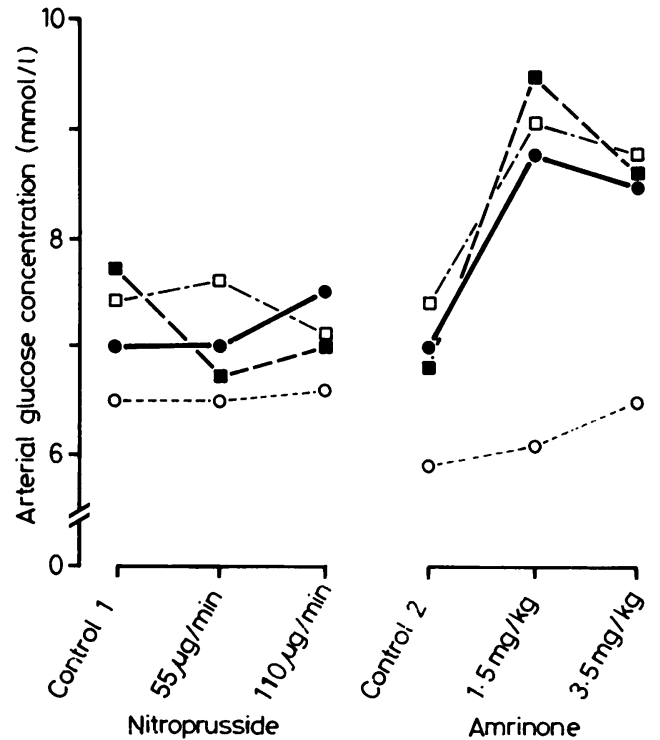

Fig. 1 Effect of nitroprusside and amrinone on arterial glucose concentrations in two patients with dilated cardiomyopathy $(\mathrm{O}$ and $\bigcirc)$ and two with coronary artery disease ( $\square$ and $\square$ ).

\section{COMPARISON BETWEEN GROUPS}

The patients with dilated cardiomyopathy showed haemodynamic and metabolic responses to the two drugs which were similar to those of the patients with coronary artery disease.

\section{PLASMA AMRINONE CONCENTRATIONS}

Estimation of plasma amrinone concentration was performed on 11 plasma samples from six patients taken from both patient groups. A log-linear relation existed between plasma amrinone concentration and either the change in cardiac index $(p<0.01)$ (Fig. 2) or percentage change in cardiac index $(r=0.75, n=11$,

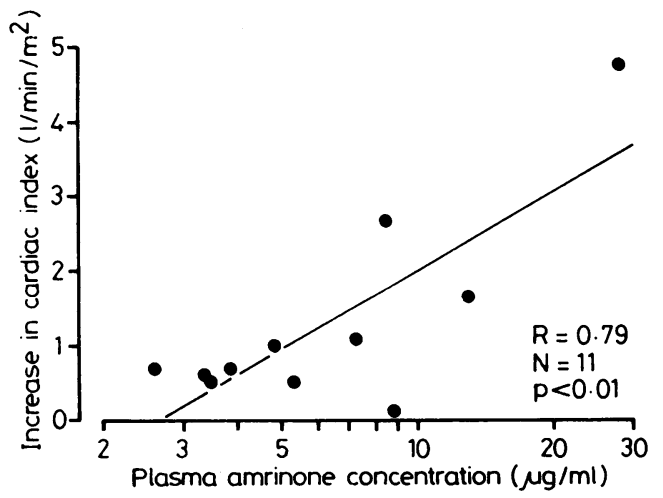

Fig. 2 Relation between the plasma amrinone concentration and the change in cardiac index. p $<0.01$ ). No relation (including non-parametric) existed between plasma amrinone concentration and changes in any contractility index measured. Similarly, no relation existed between the changes in cardiac index and changes in contractility.

No relation was found between the concentration of the metabolite, $N$-acetyl amrinone, and any alteration in haemodynamics.

\section{RELATION BETWEEN CHANGES IN \\ HAEMODYNAMICS AND METABOLISM}

In some individual patients reductions in $\max \mathrm{dP} / \mathrm{dt}$ (and other contractility indices) occurred after amrinone. The greatest reduction in $\max \mathrm{dP} / \mathrm{dt}$ after amrinone was $25 \%$ and changes in $\max \mathrm{dP} / \mathrm{dt}$ by $\pm 15 \%$ occurred after nitroprusside. We therefore arbitrarily considered that any alteration in $\max \mathrm{dP} / \mathrm{dt}$ by $\pm 20 \%$ might be due to random variation even if the drug had no positive inotropic effect. Increases in $\max \mathrm{dP} / \mathrm{dt}$ by more than $20 \%$ did occur in four patients after amrinone, but in each case arterial free fatty acid concentration also rose considerably (43$129 \%$ ). Only one other patient showed a similar increase in arterial free fatty acid concentration, but had a slight reduction in $\max \mathrm{dP} / \mathrm{dt}$. In this patient the rise in arterial free fatty acid concentration was associated with a pronounced reduction in left ventricular filling and systolic pressures. There appeared to be a relation between the changes in arterial free fatty acid

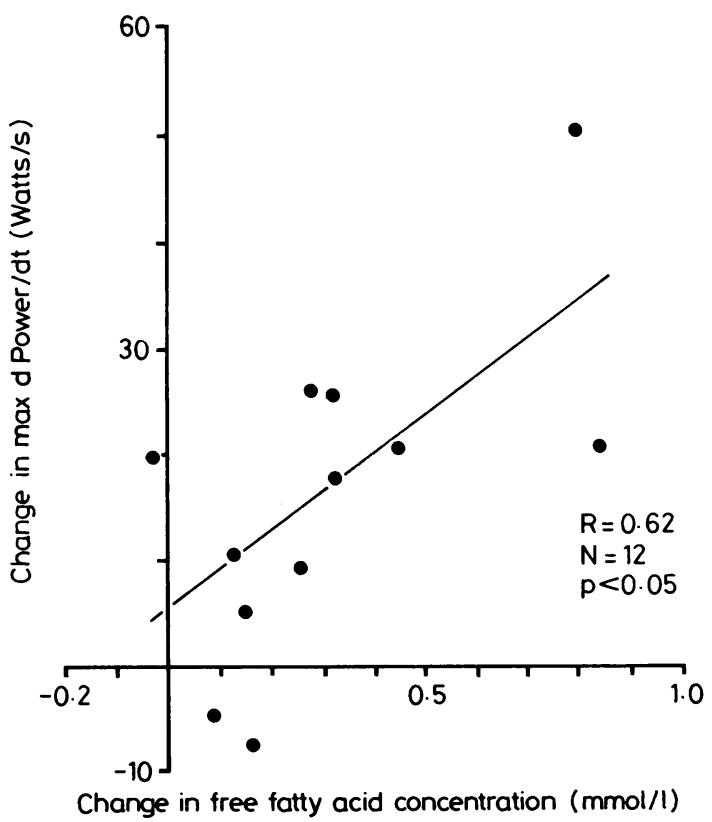

Fig. 3 Relation between the change in arterial free fatty acid concentration and the change in max dPower/dt after amrinone administration. 
concentration and changes in contractility indices, which was most obvious when max dPower/dt was the contractility index chosen (Fig. 3). No relation (including non-parametric) could be found between the change in lactate extraction after amrinone and corresponding changes in arterial free fatty acid concentrations or changes in contractility.

\section{EFFECT OF CARRIER SOLUTION}

The effect of the carrier solution was studied in seven patients with left ventricular ejection fractions between 0.19 and 0.65 (mean 0.38 ). The carrier solution did not alter heart rate, right atrial, pulmonary arterial, left atrial, left ventricular, or aortic pressures, cardiac index, or any index of contractility. In selected patients thought to be able to tolerate a lengthened procedure the effects of the carrier solution on left ventricular volumes derived angiographically or left ventricular metabolism (myocardial oxygen consumption, metabolite extraction ratios, etc) or both were determined and it was without measurable effect.

\section{BIOASSAY OF AMRINONE AMPOULES}

Each batch of amrinone solution used was bioassayed and found to have positive inotropic properties in the guinea pig myocardium in a manner previously described. ${ }^{13}$

\section{Discussion}

The resting (control) haemodynamic values, arterial concentrations, and myocardial extractions of metabolites in the patients with dilated cardiomyopathy found in this study were similar to those previously described.26 Similar resting haemodynamic and myocardial metabolic data were found in the patients with coronary artery disease and comparably poor left ventricular function. The effects of nitroprusside on haemodynamic indices and myocardial metabolism in both our groups of patients were similar to those described previously in patients with left ventricular failure. ${ }^{28}$

In general terms, in both groups of patients amrinone produced changes in haemodynamic indices similar to those seen with the pure vasodilator, nitroprusside. In particular there was no clear evidence that amrinone had a dose related, direct positive inotropic effect. A slight reduction in $\max \mathrm{dP} / \mathrm{dt}$ with nitroprusside and a slight increase with amrinone were seen, but the changes were not statistically significant and probably merely reflect the effect of slight alterations in heart rate (not significant) seen with the drugs. ${ }^{622}$ The mean slight increase in max $\mathrm{dP} / \mathrm{dt}$ after amrinone may also be, in part, the result of an increase in $\max \mathrm{dP} / \mathrm{dt}$ by more than $20 \%$ in four patients in whom arterial free fatty acid also rose appreciably. There are, of course, difficulties in using $\max \mathrm{dP} / \mathrm{dt}$ as an index of contractility in the presence of changes in heart rate, preload, and afterload, as we have previously discussed. ${ }^{6} \mathrm{~A}$ number of other contractility indices less affected by loading changes ${ }^{21-23}$ were, however, also unaltered by either amrinone or nitroprusside. Despite finding a relation between log plasma amrinone concentration and change in cardiac index, which was similar to that reported by others, ${ }^{829}$ we were unable to show a relation between plasma drug concentration and change in any contractility index. The concentration of amrinone after intravenous dosing which produced a $50 \%$ increase in cardiac index in this study was $4.4 \mu \mathrm{g} / \mathrm{ml}$ compared with 3.7 and $4.0 \mu \mathrm{g} / \mathrm{ml}$ reported by others. ${ }^{8} 29$

There are several reasons why we might have failed to confirm the increase in max $\mathrm{dP} / \mathrm{dt}$ after amrinone administration which has been described by other workers. ${ }^{3-5}$ Bioassay of the amrinone ampoules and both in vitro ${ }^{13}$ and in vivo experiments using the carrier solution, however, exclude the possibility of faults in the composition of the amrinone ampoules supplied to us or a negative inotropic effect of the carrier medium. Other workers ${ }^{19}$ have suggested that the timing of coronary arteriography, 40 minutes before our drug studies, may have been responsible for our previous failure to show an increase in max $\mathrm{dP} / \mathrm{dt}$ after amrinone. ${ }^{6}$ In the present study no difference in response to amrinone was found whether or not the patients underwent coronary arteriography before or after the drug study. In the previous studies of the effect of amrinone on contractility in patients with cardiac failure ${ }^{3-6}$ the patient groups studied were heterogeneous for aetiology of heart failure. It is possible that amrinone could have had different effects on contractility in patients with different disease processes so that the overall effects seen would have depended on the composition of the group. Our observation that in two homogeneous groups of patients with heart failure, the effects of amrinone were similar does not support this view. The probable reason that we failed to confirm the increase in contractility after amrinone which has been claimed in some small series ${ }^{3-5}$ was that those studies, unlike our own, were biased towards demonstration of a positive effect by their experimental design. They did not attempt to demonstrate a dose-response relation. Instead, the authors gave increasing doses of the drug until a haemodynamic endpoint was reached, when measurements were made. The results thus obtained were affected more by the choice of the endpoint than by the effects of the drug studied.

Nitroprusside and amrinone had similar effects on myocardial metabolism in the two groups of patients, although some differences in peripheral blood con- 
centrations of metabolites were detected. This suggests that any differences between the metabolic effects of amrinone and nitroprusside are entirely extracardiac. It was observed that the arterial concentration of lactate tended to fall with nitroprusside but not with amrinone, whereas with amrinone arterial free fatty acid and glycerol concentrations tended to rise. These changes were most pronounced in a few individual patients in whom $\max \mathrm{dP} / \mathrm{dt}$ rose after amrinone but not after nitroprusside. Similarly, arterial glucose concentration rose after amrinone in the four patients in whom it was measured. This is strong indirect evidence that amrinone causes release of catecholamines, which should increase arterial lactate, glucose, free fatty acid, and glycerol concentrations. ${ }^{30}$

Amrinone is known to possess phosphodiesterase inhibitory activity as well as an in vitro pharmacological profile similar to theophylline. ${ }^{31}{ }^{32}$ Most phosphodiesterase inhibitors cause release of catecholamines and hence increase circulating free fatty acids. ${ }^{33} \mathrm{~A}$ major part of the positive inotropic effects seen in vivo with phosphodiesterase inhibitors is the result of this catecholamine release. ${ }^{34}$ It is unlikely that phosphodiesterase inhibitors per se can increase arterial free fatty acids since the effect of cyclic adenosine monophosphate analogues is to reduce circulating free fatty acids. ${ }^{35}$ Thus the increase in free fatty acid concentration after amrinone was probably secondary to catecholamine release. Furthermore, other workers ${ }^{36}$ have found that in subjects with coronary artery disease but normal left ventricular function amrinone increased plasma concentrations of adrenaline from 0.29 to $0.39 \mathrm{ng} / \mathrm{ml}$ and of noradrenaline from 0.79 to $1.0 \mathrm{ng} / \mathrm{ml}$. These changes in mean values were not statistically significant, but the authors did not quote standard deviations or standard errors, which must have been large. It is clear that after administration of amrinone some of the patients must have experienced a considerable increase in circulating catecholamines. It certainly does not support the view, expressed be some, ${ }^{2}$ that amrinone's vasodilator properties may be due to "withdrawal of enhanced sympathetic constrictor tone consequent to improvement in ventricular performance."

If, as we suspect, it is subsequently confirmed that amrinone does cause release of catecholamines and increase of circulating free fatty acids in some individuals this may provide a mechanism for the increased incidence of ventricular arrhythmias and death in patients receiving amrinone reported by some groups of workers. ${ }^{153} 37$

Some workers have found that amrinone acutely increases exercise performance. ${ }^{38}$ Since acute administration of vasodilators do not do this, they have concluded that amrinone's additional effect must be the result of improved cardiac performance. This suggestion requires many unjustified assumptions and neglects the possibility that reduction in muscle fatigue could be due to a direct phosphodiesterase inhibitory action on skeletal muscle, ${ }^{39}$ or an indirect effect via catecholamine release. ${ }^{40}$

In chronic cardiac failure drugs which increase intracellular cyclic adenosine monophosphate do not appear to have positive inotropic effects, whether they are adenyl cyclase activators (for example, glucagon) ${ }^{4-43}$ or phosphodiesterase inhibitors (for example, amrinone)..$^{12} 13 \mathrm{In}$ this regard it is of interest that the cyclic adenosine monophosphate analogue, dibutyryl cyclic adenosine monophosphate, has similar properties to amrinone, producing large increases in contractility in normal myocardium,${ }^{44}$ whereas in chronic cardiac failure the improvements in cardiac index and filling pressures seen ${ }^{4546}$ are the result of vasodilatation without any evidence of an increase in contractility. ${ }^{46}$

We conclude that in patients with chronic cardiac failure amrinone acts by vasodilatation, similar to that produced by nitroprusside, with no evidence of a direct effect on contractility or the myocardium. In a few individual patients improvements in contractility were probably the result of catecholamine release.

We thank C M Miles, J L Evans, and R Islam for their technical assistance.

The research was supported by grants from the Endowment Committee and the Cardiac Research Fund at St Thomas's Hospital.

\section{References}

1 LeJemtel TH, Keung E, Sonnenblick EH, et al. Amrinone: a new non-glycosidic, non-adrenergic cardiotonic agent effective in the treatment of intractable myocardial failure in man. Circulation 1979; 59: 1098-104.

2 LeJemtel TH, Keung EC, Schwartz WJ, et al. Hemodynamic effects of intravenous and oral amrinone in patients with severe heart failure: relationship between intravenous and oral administration. Trans Assoc Am Physicians 1979; 92: 325-33.

3 Benotti JR, Grossman W, Braunwald E, Davolos DD, Alousi AA. Hemodynamic assessment of amrinone: a new inotropic agent. $N$ Engl f Med 1978; 299: 1373-7.

4 Cárdenas LM, Vidaurri DA. Estudio de los efectos hemodinámicos de diferentes dosis de un nuevo inótropico: la amrinona. Arch Inst Cardiol Mex 1979; 49: 961-8.

5 Jennings K, Gwilt D, Crean P, Turnbull S, Gold R, Julian DG. The clinical cardiovascular pharmacology of amrinone. A selective cardiotonic agent. Acta Cardiol (Brux) 1982; suppl 28: 67-75.

6 Wilmshurst PT, Thompson DS, Jenkins BS, Coltart DJ, WebbPeploe MM. Haemodynamic effects of intravenous amrinone in patients with impaired left ventricular function. Br Heart $\mathcal{f} 1983$; 49: 77-82.

7 Onuaguluchi G, Tanz RD. Cardiac effects of amrinone on rabbit papillary muscle and guinea-pig Langendorff heart preparations. f Cardiovasc Pharmacol 1981; 3: 1342-55. 
8 Alousi AA, Edelson J. Amrinone. In: Goldberg ME, ed. Pharmacological and biochemical properties of drug substances. Vol 3. Washington DC: American Pharmaceutical Association, 1981: 120-47.

9 Wilmshurst PT, Webb-Peploe MM. Side effects of amrinone therapy. Br Heart $\mathcal{f}$ 1983; 49: 447-51.

10 Bayliss J, Norell M, Canepa-Anson R, Reuben SR, Poole-Wilson PA, Sutton GC. Acute haemodynamic comparison of amrinone and pirbuterol in chronic heart failure. Additional effects of isosorbide dinitrate. $\mathrm{Br}$ Heart $\mathrm{f}$ 1983; 49: 214-21.

11 Wilmshurst PT, Al-Hasani SFA, Semple MJ, et al. The effects of amrinone on platelet count, survival and function in patients with congestive cardiac failure. Br 7 Clin Pharmacol 1984; 17: 317-24.

12 Gaide MS, Fitterman WS, Wiggins JR, Myerburg RJ, Cameron JS, Bassett AL. Amrinone relaxes potassium-induced contracture of failing right ventricular muscle of cats. $\mathcal{F}$ Cardiovasc Pharmacol 1983; 5: 335-40.

13 Wilmshurst PT, Walker JM, Fry CH, et al. The inotropic and vasodilator effects of amrinone on isolated human tissue. Cardiovasc Res (In press).

14 Dunkman WB, Wilen MM, Franciosa JA. Adverse effects of long-term amrinone administration in congestive heart failure. Am Heart f 1983; 105: 861-3.

15 Leier CV, Dalpiaz K, Huss P, et al. Amrinone therapy for congestive heart failure in outpatients with idiopathic dilated cardiomyopathy. Am $\mathcal{F}$ Cardiol 1983; 52: 304-8.

16 Baim DS, McDowell AV, Cherniles J, et al. Evaluation of a new bipyridine inotropic agent - Milrinone - in patients with severe congestive heart failure. $N$ Engl $\mathcal{F}$ Med 1983; 309: 748-56.

$17 \mathrm{Col} \mathrm{J}$, Cheron P, Jouret G, Harvengt C, Petein M. Haemodynamic effects of AR-L115 BS in severe chronic congestive heart failure [Abstract]. Eur $\mathcal{F}$ Clin Invest 1982; 12 (suppl): $47 \mathrm{~S}$.

18 Thompson DS, Naqvi N, Juul SM, Coltart DJ, Jenkins BS, Webb-Peploe MM. Haemodynamic and metabolic effects of atenolol in patients with angina pectoris. $\mathrm{Br}$ Heart $\mathcal{F}$ 1980; 43: 668-79.

19 Timmis A, Daly K, Jewitt DE. Haemodynamic effects of intravenous amrinone in patients with impaired left ventricular function [Letter]. Br Heart $\mathcal{F}$ 1983; 50: 106-8.

20 Brooksby IAB, Swanton RH, Jenkins BS, Webb-Peploe MM. Long sheath technique for introduction of catheter tip manometer or endomyocardial bioptome into left or right heart. $\mathrm{Br}$ Heart $\mathrm{f}$ 1974; 36: 908-12.

21 Veragut UP, Krayenbühl HP. Estimation and quantification of myocardial contractility in the closed-chest dog. Cardiologia 1965; 47: 96-112.

22 Yang SS, Bentivoglio LG, Maranhão V, Goldberg H. From cardiac catheterization data to hemodynamic parameters. 2nd ed. Philadelphia: FA Davis, 1978: 233-358.

23 Kolettis M, Jenkins BS, Webb-Peploe MM. Assessment of left ventricular function by indices derived from aortic flow velocity. Br Heart 7 1976; 38: 18-31.

24 Stein PD, Sabbah HN. Rate of change of ventricular power: an indicator of ventricular performance during ejection. Am Heart $\mathcal{f}$ 1976; 91: 219-27.

25 Keilin D, Hartree EF. Properties of glucose oxidase (Notatin) Biochem f 1948; 42: 221-9.

26 Thompson DS, Naqvi N, Juul SM, et al. Cardiac work and myocardial substrate extraction in congestive cardiomyopathy. $\mathrm{Br}$ Heart f 1982; 47: 130-6.

27 Kullberg MP, Dorrbecker B, Lennon J, Rowe E, Edelson J. High performance liquid chromatographic analysis of amrinone and its $\mathrm{N}$-acetyl derivative in plasma: pharmacokinetics of amrinone in the dog. 7 Chromatogr 1980; 187: 264-70.

28 Thompson DS, Juul SM, Wilmshurst $\mathrm{P}$, et al. Effects of sodium nitroprusside upon cardiac work, efficiency and substrate extraction in severe left ventricular failure. Br Heart $\mathcal{F}$ 1981; 46: 394 400.

29 Edelson J, LeJemtel TH, Alousi AA, Biddlecome CE, Maskin :CS, Sonnenblick EH. Relationship between amrinone plasma $\overrightarrow{\bar{S}}$ concentration and cardiac index. Clin Pharmacol Ther 1981; 29: 723-8.

30 Weiner $\mathbf{N}$. Norepinephrine, epinephrine and the sympathomimetic amines. In: Gilman AG, Goodman LS, Gilman A, ed. Goodman and Gilmans the pharmacological basis of therapeutics. 6th ed. New York: MacMillan, 1980: 138-75.

31 Honeriäger $P$, Schafer-Korting $M$, Reiter $M$. Involvement of \& cyclic AMP in the direct inotropic action of amrinone: biochemical and functional evidence. Naunyn Schmiedebergs Arch Pharmacol 1981; 318: 112-20.

32 Endoh M, Yamashita S, Taira N. Positive inotropic effect of $\vec{\omega}$ amrinone in relation to cyclic nucleotide metabolism in the canine ventricular muscle. $\mathcal{O}$ Pharmacol Exp Ther 1982; 221: 775-83.

33 Vestal RE, Eiriksson CE Jr, Musser B, Ozaki LK, Halter JB. or Effect of intravenous aminophylline on plasma levels of ? catecholamines and related cardiovascular and metabolic $\vec{\omega}$ responses in man. Circulation 1983; 67: 162-71.

34 Marcus ML, Skelton CL, Grauer LE, Epstein SE. Effects of $\bigcirc$ theophylline on myocardial mechanics. Am $\mathcal{F}$ Physiol 1972; 222: $1361-5$.

35 Hashimoto H, Okumura K, Niizoe K, Ogawa K. Effects of dibutyryl cyclic AMP on patients with severe heart failure. $f_{p n}$ Heart f 1982; 23: 1021-7.

36 Saborowski F, Griebenow R, Sirinyan G, Hense K. Metabolische und hämodynamische Befunde nache Gabe von Amrinone bei ${ }^{+}$ Patienten mit koronarer Herzkrankheit. Z Kardiol 1983; 72 (suppl 1): 81 .

37 Kinney EL, Carlin B, Ballard JO, Burks JM, Hallahan WF, Zelis R. Clinical experience with amrinone in patients with advanced congestive heart failure. 7 Clin Pharmacol 1982; 22: 433-40.

38 Siskind SJ, Sonnenblick EH, Forman R, Scheuer J, LeJemtel Ф TH. Acute substantial benefit of inotropic therapy with amrinone on exercise hemodynamics and metabolism in severe congestive heart failure. Circulation 1981; 64: 966-73.

39 Foltz E, Ivy AC, Barborka CJ. The use of double work periods in the study of fatigue and the influence of caffeine on recovery. Am f Physiol 1942; 136: 79-86.

40 Bowman WC, Nott MW. Actions of sympathomimetic amines and their antagonists on skeletal muscle. Pharmacol Rev 1969; 21: 27-72.

41 Vander Ark CR, Reynolds EW Jr. Clinical evaluation of glucagon by continuous infusion in the treatment of low cardiac output states. Am Heart f 1970; 79: 481-7.

42 Armstrong PW, Gold HK, Daggett WM, Austen WG, Sanders CA. Hemodynamic evaluation of glucagon in symptomatic heart disease. Circulation 1971; 44: 67-73.

43 Goldstein RE, Skelton CL, Levey GS, Glancy DL, Beiser GD, $>$ Epstein SE. Effects of chronic heart failure on the capacity of glucagon to enhance contractility and adenyl cyclase activity of
human papillary muscles. Circulation $1971 ; 44: 638-48$.

44 Skelton CL, Levey GS, Epstein SE. Positive inotropic effects of dibutyryl cyclic adenosine $3^{\prime}, 5^{\prime}$-monophosphate. Circ Res 1970; 26: 35-43.

45 Matsui S, Murakami E, Takekoshi N, Emoto J, Matoba M. O Hemodynamic effects of dibutyryl cyclic AMP in congestive heart failure. Am $\mathcal{F}$ Cardiol 1983; 51: 1364-8.

46 Satoh K. Effect of dibutyryl cyclic AMP on hemodynamics and $\overparen{D}$ metabolism of carbohydrates and lipid after extracorporeal circu-? lation in cardiac surgical patients [In Japanese with English Abstract]. Kyobu Geka 1980; 28: 425-37. 